\title{
Spontaneous intracorneal haemorrhage
}

\author{
$R$ L S YEOH, N COX, AND M G FALCON \\ From the Ophthalmic Department, St Thomas's Hospital, London SE1 7EH
}

SUMMARY We describe the unusual complication of spontaneous intracorneal haemorrhage in a patient who used an extended-wear contact lens. This was severe enough to cause corneal blood staining and ulceration which required surgical intervention.

A 65-year-old male Caucasian bricklayer who was otherwise fit and who had had no previous eye disease underwent an uneventful intracapsular cataract extraction through a standard limbal incision to the left eye in October 1979 and to the right eye in February 1981 . He was subsequently fitted with $30 \%$ Hydrogel daily wear contact lenses in June 1981 and achieved visual acuities of $6 / 9$ (left) and 6/6 (right). Unfortunately he often failed to attend for treatment and also misused these lenses by wearing them on an extended-wear basis. This, together with lens spoilation due to lipid deposition, became a problem, and he was subsequently fitted with $60 \%$ Hydrogel lenses in March 1982 for use on an extended-wear basis.

He presented in April 1982 complaining of painful blurring of vision in the right eye for three days. His vision had dropped from $6 / 6$ to counting fingers, and he was found to have a central superficial corneal ulcer with a little stromal ulceration associated with a brisk anterior uveitis but no hypopyon. A frond of deep corneal vessels at the level of Descemet's membrane was noted at 9 o'clock, away from the previous limbal section and extending half way to the visual axis. He was admitted to hospital with a diagnosis of suppurative keratitis. Microbiological investigation, including superficial corneal scraping, revealed no organisms, but he was treated with an atraumatic subconjunctival injection of $20 \mathrm{mg}$ gentamicin and Mydricaine no. 2 (atropine sulphate, procaine, adrenaline solution) followed by intensive topical antibiotics and mydriatics.

The following day the eye was more comfortable and clinically much quieter, but he had developed a small intracorneal haemorrhage in the posterior third of the corneal stroma arising at the temporal limbus and extending $2 \mathrm{~mm}$ towards the visual axis. Over the next few hours this gradually enlarged, with an obvious horizontal blood level, but this progression

Correspondence to Mr M G Falcon, FRCS. halted when approximately $4 \mathrm{~mm}$ from the limbus, leaving the visual axis unaffected. Two days later the ulcer, uveitis, and infiltration had almost cleared, but he then developed a further secondary intrastromal haemorrhage which obscured the visual axis reducing the vision to hand movements only.

Investigations revealed a normal blood profile and no bleeding disorder. At no time during this episode did he have blood in the anterior chamber, any increase in intraocular pressure, or any significant stromal oedema.

Over the next few months the eye remained quiet, with a visual acuity of hand movements only, but the corneal stroma developed diffuse central and inferotemporal blood staining. In July 1982 he developed pain and watering in the right eye and was found to have an area of central corneal stromal breakdown and very deep ulceration over the blood stained area (Fig. 1). The iron-derived pigment in the ulcer base

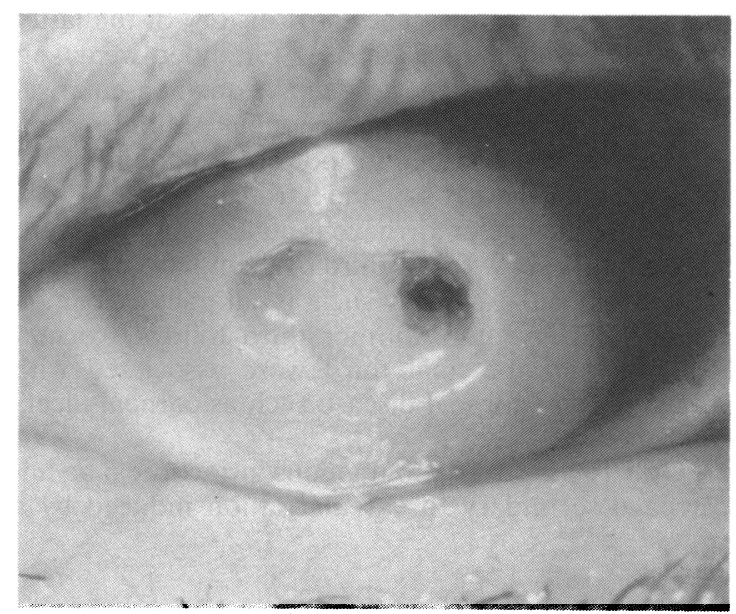

Fig. 1 Blood stained cornea with deep central corneal ulcer, a part of which is pigmented. 
looked suspiciously like iris, but there was no leak, the anterior chamber was deep, and the pupil was round.

He was treated with chloramphenicol drops four times a day, but the ulcer enlarged and deepened. A bandage contact lens was not tolerated. Because the corneal pathology was purely stromal and there had been no suggestion of endothelial compromise, and because the disease had by now extended to the limbus, an $8 \mathrm{~mm}$ diameter, deep lamellar keratoplasty was carried out in September 1982. At operation he was found to have a large pool of gelatinous altered blood between the stroma and Descemet's membrane. This was removed together with the blood stained cornea, and a pre-Descemet's lamellar graft was carried out. A small amount of residual blood remained. Postoperatively he achieved a visual acuity of $6 / 9$ with a gas permeable daily-wear contact lens. Good vision with the contact lens has continued to date.

Endothelial specular photographs taken a month after grafting showed enlarged but plentiful endothelial cells centrally with some variation in size. This was felt to be consistent with an otherwise uneventful cataract extraction and supported the choice of lamellar keratoplasty rather than penetrating keratoplasty in this case.

\section{Discussion}

This patient had two unusual corneal problems: (1) spontaneous intracorneal haemorrhage; (2) corneal blood staining without endothelial involvement leading to ulceration and requiring lamellar keratoplasty.

The intracorneal haemorrhage arose from deep stromal vascularisation, which was the result either of using the extended-wear soft contact lens or suppurative keratitis. The short history of the latter makes this an unlikely cause. Extended-wear soft contact lenses can lead to deep stromal vascularisation, but superficial corneal vascularisation is commoner. ${ }^{1}$ It has also been reported in patients using cosmetic, daily-wear soft and hard lenses. ${ }^{2}$

Intracorneal haemorrhage is an uncommon event. The reports show that a third of them have occurred after intraocular surgery such as cataract extraction or corneal grafting; another third followed ocular trauma; and the final third were associated with inflammatory corneal disease such as corneal ulcers and chemical burns. ${ }^{3}$

Only two cases of spontaneous intracorneal haemorrhage secondary to vascularisation induced by a contact lens have been reported..$^{45}$ Both followed the use of daily-wear soft contact lenses in aphakic eyes and both were subepithelial haemorrhages. The first cleared spontaneously in three weeks, the second was extensive and required debridement. The haemorrhage in our patient occurred at a much deeper level and was severe enough to cause extensive stromal blood staining and subsequent necrosis and ulceration of a large area of stroma, which required lamellar keratoplasty. Recently Ormerod and Egan described three cases of intracorneal haemorrhage secondary to microbial keratitis, two of which were subepithelial and cleared rapidly. The other was midstromal and pre-Descemet's and was still present after one month.

Corneal blood staining classically follows traumatic hyphaemas where there has been severely raised intraocular pressure and compromised endothelial function.' But cases have been reported of staining occurring in patients with traumatic hyphaemas and low intraocular pressures. ${ }^{8}$ The mechanism postulated in these cases was that the staining was secondary to contusion necrosis of the endothelium or to temporary endothelial decompensation in the presence of significant hyphaemas. Corneal blood staining in our case occurred as a direct result of haemorrhage within the cornea. Corneal blood staining generally clears spontaneously, though it may take up to three years. ${ }^{8}$ For reasons that are not clear this patient developed stromal necrosis and ulceration.

We are grateful to Mr B Cobb, FRCS, consultant ophthalmologist, Greenwich District Hospital, for referring the patient and allowing us to publish this report.

\section{References}

1 Dohlman C. Complications in therapeutic soft lens wear. Ophthalmology 1974; 78: OP 399.

2 Nirankari VS, Karesh J, Lakhanpal V, Richards RD. Deep stromal vascularisation associated with continuous daily wear contact lenses. Arch Ophthalmol 1983; 101: 46-7.

3 Meuenzler W. Intracorneal haemorrhage. Am J Ophthalmol 1973; 76: 440-2.

4 Montalbo A, Lambros J. Corneal subepithelial haemorrhage. Arch Ophthalmol 1977; 95: 702-3.

5 Hurwitz BS. Spontaneous intracorneal haemorrhage caused by aphakic contact lens wear. Ann Ophthalmol 1981; 13: 57-9.

6 Ormerod LD, Egan KM. Spontaneous hyphaema and corneal haemorrhage as complications of microbial keratitis. $\mathrm{Br} J$ Ophthalmol 1987; 71: 933-7.

7 Brodrick JD. Corneal blood staining after hyphaemas. $\mathrm{Br} J$ Ophthalmol 1972: 56: 589-92.

8 Beyer TL, Hirst LW. Corneal blood staining at low pressures. Arch Ophthalmol 1985; 103: 654-5.

Accepted for publication 11 August 1988. 